\title{
Detecção de Distúrbios em Qualidade de Energia usando modelos NARX Neurais
}

\author{
Isaque Fernando Pereira Alcântara* \\ Bruno Henrique Groenner Barbosa** \\ Danton Diego Ferreira** \\ * Programa de Pós-graduação em Engenharia de Sistemas e \\ Automação, Universidade Federal de Lavras, MG, (e-mail: \\ ifpalc@gmail.com). \\ ** Departamento de Automática, Universidade Federal de Lavras, MG, \\ (e-mails: brunohb@ufla.br,danton@ufla.br)
}

\begin{abstract}
The study of the area of Power Quality (PQ) has grown recently. The increasing use of high power converters and of nonlinear loads cause changes in the electrical signal (current and voltage), which are often called disturbances. This work presents a system to detect disturbances of PQ based on a neural NARX model (Nonlinear Autoregressive with eXogenous inputs). From the application of a notch filter on the voltage signal, the neural network estimates the signal value one step ahead and, through the residue analysis of this model, the disturbance is detected. The system was tested on simulations of six classes of disturbances, with different signal-to-noise ratios and variations of the fundamental frequency, showing a very high detection accuracy using few samples of the event with disturbance.

Resumo: O estudo da área de Qualidade de Energia Elétrica (QEE) tem crescido muito nos últimos anos. O aumento no uso de conversores de alta potência e de cargas não lineares com potência elevada causam perturbações no sinal elétrico (corrente e tensão), chamadas de distúrbios. Este trabalho apresenta um sistema de detecção de distúrbios de qualidade da energia elétrica (QEE) baseado em um modelo neural NARX (Nonlinear Autoregressive with eXogenous inputs). A partir da aplicação de um filtro notch no sinal de tensão, a rede neural estima o valor do sinal um passo à frente e, por meio da análise de resíduo deste modelo, o distúrbio é detectado. O sistema foi testado em simulações de seis classes de distúrbios, com diferentes relações sinal-ruído e variações na frequência fundamental, apresentando um acerto de detecção bastante elevando utilizando poucas amostras do evento com distúrbio.
\end{abstract}

Keywords: Detection; NARX models; Neural Networks; Power Quality; Electrical Disturbance. Palavras-chaves: Detecção; Modelos NARX; Redes Neurais; Qualidade de Energia; Distúrbios.

\section{INTRODUÇÃO}

A Qualidade da Energia Elétrica (QEE), ou Power Quality $(P Q)$, estuda as variações em regime permanente que provocam desequilíbrio no sinal de tensão ou de corrente causadas por falhas na rede elétrica (Ghosh and Ledwich, 2012). O estudo desses fenômenos em QEE iniciou-se na década de 90, evidenciando a crescente introdução de cargas não lineares nas malhas elétricas, como o estudo de Ward et al. (1990) e as normas IEEE Standards Board (1995) e IEC TR 61000-2-1 (1990), que caracterizaram distúrbios como as elevações (swells) e afundamentos (sags) de tensão, harmônicos, inter-harmônicos, interrupções, transitórios, notches e suas combinações (Barbosa and Ferreira, 2013).

As causas dos distúrbios em QEE podem ser as fontes chaveadas, as descargas atmosféricas, a sobrecarga de equipamentos ligados simultaneamente, o chaveamento de banco de capacitores, curtos circuitos, má operação da rede, o uso de fontes de geração distribuída, entre outras (Ribeiro et al., 2018).
As consequências da queda da qualidade da energia elétrica podem ser desde a queima de eletrônicos, a inoperância de equipamentos, perda do conjugado de motores, decaimento do fator de potência, o aquecimento de condutores (em especial o neutro) e a queima de fusíveis até a perda de computadores (Bollen and $\mathrm{Gu}, 2006$ ).

Para a solução dos problemas de QEE, as redes inteligentes em sistemas de distribuição, nominadas de Smart Grids, são apontadas como uma boa opção (Brown, 2008). Isso ocorre graças ao poder das ferramentas de inteligência computacional (IC), integráveis em Smart Grids, que possuem a capacidade de detectar os distúrbios elétricos. A detecção deve ser realizada por meio de sistemas de baixa complexidade computacional, permitindo assim o monitoramento em tempo real para aplicações de compensação e proteção (Ribeiro et al., 2018).

Normalmente, esses sistemas possuem como etapas a extração de parâmetros e a aplicação de um algoritmo que realize a detecção baseada nos parâmetros extraídos. A extração de parâmetros consiste na transformação dos da- 
dos (normalmente janelas de dados) para um novo espaço onde as diferenças entre os distúrbios e os sinais nominais ficam destacadas. Estas características, ou parte delas, são então utilizadas em um sistema de detecção. Seguindo essa abordagem, vários sistemas de detecção de distúrbios têm sido propostos (Ribeiro et al., 2018). Dentre eles destacamse aqueles com diferentes ferramentas para extração de características, como a Transformada de Wavelets (Thirumala et al., 2018), as Estatísticas de Ordem Superior (Barbosa and Ferreira, 2013), a Análise de Componentes Independentes (Nagata et al., 2018), e aqueles com diferentes técnicas de detecção, baseados principalmente em Máquinas de Vetor de Suporte (SVM) (Thirumala et al., 2018), Árvores de Decisão (Achlerkar et al., 2018; Barbosa and Ferreira, 2013), Classificadores Bayesianos (Barbosa and Ferreira, 2013; Ribeiro et al., 2018) e Redes Neurais Artificiais (Kamble and Dupare, 2014). Uma boa revisão de métodos de detecção de eventos de QEE pode ser encontrada em (Mahela et al., 2015).

Nos métodos de detecção encontrados na literatura, é comum a análise de uma janela de dados para que a detecção ou não do distúrbio seja realizada. Por exemplo, Mendes et al. (2015) conseguiram detectar distúrbios utilizando ciclos completos de 256 amostras, por meio da análise multidimensional dos dados e com baixo custo computacional. Da mesma forma, Barbosa and Ferreira (2013); Ribeiro et al. (2018) utilizaram 4 ciclos de 256 amostras para detectar e classificar distúrbios. No entanto, é desejável que um sistema de detecção perceba a alteração na QEE assim que um distúrbio ocorra, para que medidas que mitiguem seus efeitos sejam aplicadas o quanto antes.

Isto posto, o objetivo deste trabalho é propor um detector baseado em modelo neural NARX (Nonlinear Autoregressive with eXogeneous input) feedforward capaz de identificar, de forma eficiente e com poucas amostras, a presença de distúrbios que reduzem a qualidade da energia elétrica em sistemas elétricos de potência.

Para isso, a entrada do modelo neural é obtida por meio de um filtro Notch de parâmetros fixos (recuperação da própria componente fundamental quando não houver variação da frequência da mesma) e a saída é o sinal de tensão monitorado da rede elétrica. A partir da análise dos resíduos desse modelo, a detecção ou não de um distúrbio é realizada. Testes foram implementados de forma a verificar a eficácia do método em diferentes relações sinal-ruído, em variações da frequência fundamental, diferentes tipos de distúrbios e em diferentes configurações da estrutura da rede NARX (número de atrasos e de neurônios escondidos).

O artigo está organizado da seguinte forma. Na próxima seção é apresentada a formulação do problema de detecção de distúrbios elétricos. Na Seção III é apresentado o método proposto. Os resultados são apresentados na Seção IV e as conclusões na Seção V.

\section{DESCRIÇÃO DO PROBLEMA}

\subsection{Distúrbios em Qualidade de Energia}

Os distúrbios em QEE podem ser identificados no sinal discreto da tensão de $N$ elementos não sobrepostos.
Conforme o modelagem descrita por Ribeiro and Pereira (2007), o sinal de tensão $v(n)$ de $n$-ésimas amostras de $N$, onde $n=0, \ldots, N-1$, contém a componente fundamental $f(n)$, as distorções harmônicas $h(n)$, as distorções inter-harmônicas $i(n)$, os fenômenos transitórios $t(n)$ e um ruído independente, chamado $r(n)$. Este por sua vez é distribuído de forma homogênea ao longo do sinal. A série temporal $v(n)$ com as componentes do sinal de tensão é representada por:

$$
v(n)=f(n)+h(n)+i(n)+t(n)+r(n) .
$$

A componente fundamental $f(n)$ é dada por,

$$
f(n)=A_{0}(n) \cos \left[2 \pi \frac{f_{0}(n)}{f_{s}} n+\theta_{0}(n)\right]
$$

em que $A_{0}(n)$ corresponde a amplitude do sinal, $f_{0}(n)$ é a frequência fundamental e $\theta_{0}(n)$ representa a fase da componente fundamental.

A soma total dos eventos harmônicos no sinal é descrita como:

$$
h(n)=\sum_{m=1}^{M} h_{m}(n)
$$

em que $M$ é o número de componentes harmônicos presentes e

$$
h_{m}(n)=A_{m}(n) \cos \left[2 \pi m \frac{f_{0}(n)}{f_{s}} n+\theta_{m}(n)\right] \text {, }
$$

sendo que $A_{m}(n), f_{0}(n)$ e $\theta_{m}(n)$ correspondem a amplitude, frequência fundamental e fase do harmônico, respectivamente.

O total de eventos inter-harmônicos é descrito por:

$$
i(n)=\sum_{j=1}^{J} i_{j}(n)
$$

em que $J$ é o número de componentes inter-harmônicos e

$$
i_{j}(n)=A_{I, j}(n) \cos \left[2 \pi \frac{f_{I, j}(n)}{f_{s}} n+\theta_{I, j}(n)\right],
$$

com $A_{I, j}(n), f_{I, j}(n)$ e $\theta_{I, j}(n)$, nesta sequência, representando a amplitude, frequência e fase do inter-harmônico.

O componente $t(n)$ na Eq. 1, refere-se aos distúrbios transitórios, representado pela soma dos eventos impulsivos, oscilatórios e notches, conforme a equação:

$$
t(n)=t_{i m p}(n)+t_{n o t}(n)+t_{o s c}(n) .
$$

As equações 9, 10 e 11 representam os modelos matemáticos de cada tipo de evento transitório: 
Tabela 1. Tabela com classificação de distúrbios.

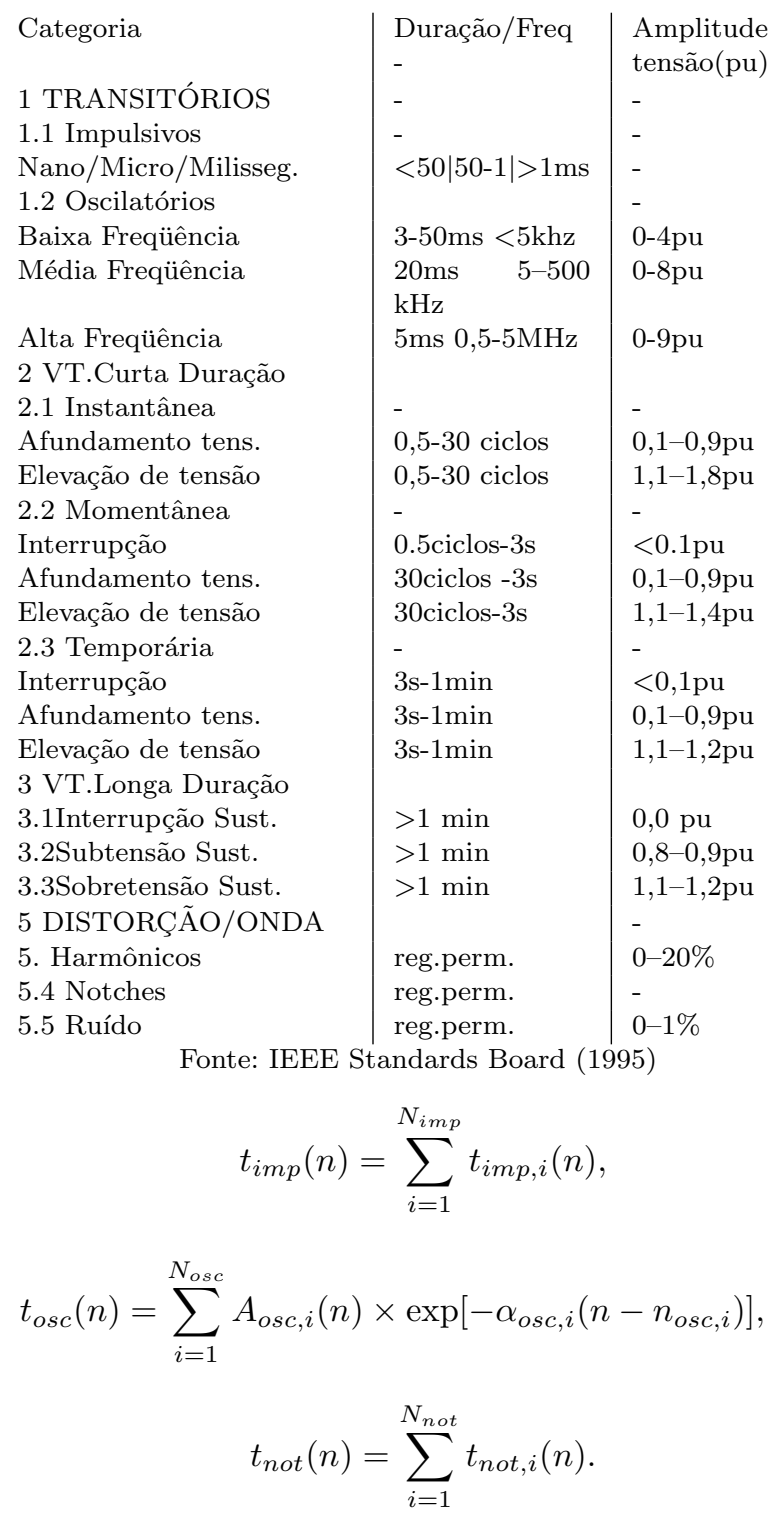

Dentre as classes eventos impulsivos, oscilatórios e notches, se encontram os distúrbios spike (impulsivo), notch (oscilatório), sag (afundamento), swell (elevação) e os flickers (flutuações de tensão que podem conter sags e swells juntos). Além disso, a classificação desses distúrbios pode ser feita conforme a sua duração, sendo considerados de curta ou longa duração. As terminologias e classificações de cada evento foram organizadas na Tab. 1 normatizada pelo IEEE Standards Board (1995).

\section{MÉTODO PROPOSTO}

De forma a detectar a presença de distúrbios no sinal de tensão, a metodologia apresentada na Fig.1 é proposta neste trabalho. As etapas desenvolvidas são apresentadas a seguir.

\subsection{Filtro Notch}

O sinal de tensão $v(n)$ foi pré-processado por meio de um filtro Notch e decomposto de acordo com as componentes

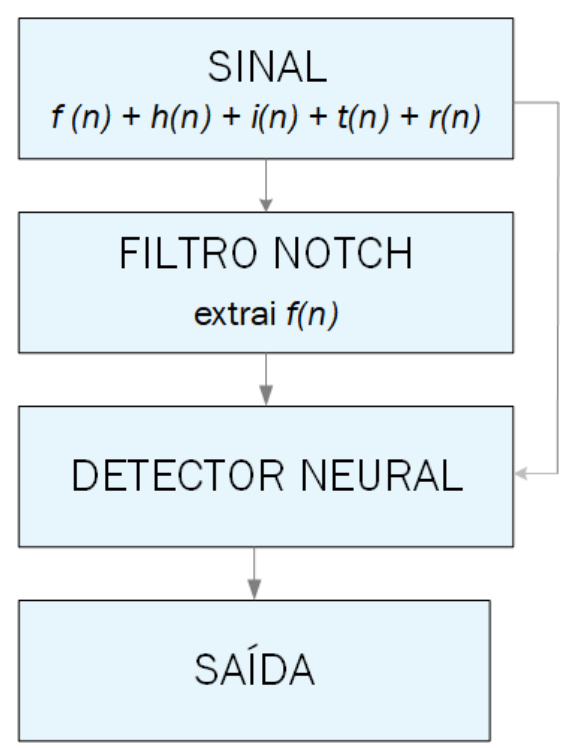

Figura 1. Método de detecção de distúrbios proposto.

presentes no sinal. A frequência de corte do filtro foi definida como $f_{0}=60 \mathrm{~Hz}$. Dessa forma, $v(n)$ é decomposto em duas variáveis: $i i$ componente fundamental $f(n)$ e $i i$. os eventuais harmônicos, inter-harmônicos, transitórios e ruído. O filtro utilizado é do tipo IIR (resposta ao impulso infinita) de segunda ordem, pois conforme Mitra and Kuo (2006) este tipo de filtro possui um baixo custo computacional e também uma boa seletividade. A transformada $Z$ deste filtro é representada por:

$$
H(z)=\frac{1+a z^{-1}+z^{-2}}{1+\rho a z^{-1}+\rho^{2} z^{-2}},
$$

em que $a=-2 \cos \omega_{0}$, e fator notch $\rho$ igual a 0,97 .

\subsection{Detector NARX Neural}

Os modelos NARX neurais são Redes Neurais Artificiais preditivas com capacidade de regressão não linear. Segundo (Siegelmann et al., 1997), os modelos NARX neurais se constituem de uma rede perceptron multicamadas (MLP), representada por uma função não-linear $f$, conforme a seguinte equação:

$$
y(k)=f\left[u(k-1), \ldots, u\left(k-n_{u}\right), y(k-1), \ldots, y\left(k-n_{y}\right)\right],
$$

em que $u\left(k-n_{u}\right)$ corresponde a entrada com $n$-ésimos atrasos e $y\left(k-n_{y}\right)$ indicam os $n$-ésimos atrasos ou estados passados do sinal de saída $y$, sendo $n_{u}$ e $n_{y}$ os maiores atrasos respectivos. A Fig. 2 apresenta a estrutura utilizada da rede, feedforward com uma única camada escondida (neurônios escondidos com função de ativação do tipo tangente hiperbólica e neurônio de saída linear), onde o número de atrasos da entrada e saída e o número de neurônios escondidos serão avaliados na próxima seção.

Para a avaliação do desempenho do modelo neural foi utilizado o Erro Médio Quadrático (MSE), descrito por:

$$
M S E=\frac{1}{N} \sum_{i=1}^{N}(y(i)-\hat{y}(i))^{2}
$$


no qual o erro (MSE) do estimador corresponde à média do quadrado do valor do sinal de tensão monitorado $y$ subtraído pelo valor estimado pela rede NARX um passo à frente, $\hat{y}$.

A partir da análise do valor do erro de predição um passo à frente da rede, quando este ultrapassar um limiar, é considerada a ocorrência de um distúrbio, uma vez que o treinamento da rede é realizado com dados de tensão nominais apenas, ou seja, sem a presença de distúrbios.

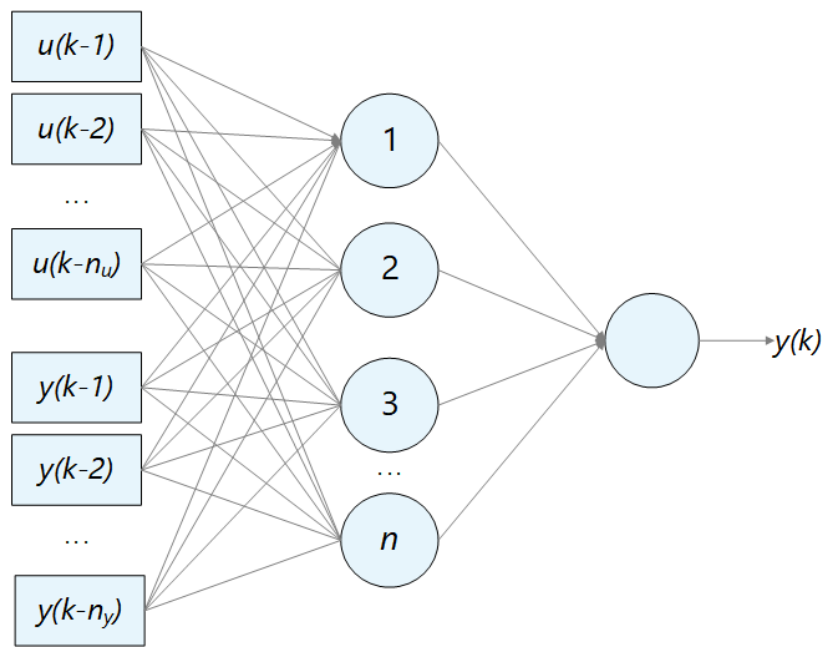

Figura 2. Rede Neural NARX utilizada como detector de distúrbios.

\section{RESULTADOS E DISCUSSÃO}

Um total de 10.000 eventos foram gerados para o caso da rede elétrica não possuir distúrbios (classe de sinais nominais de tensão), de forma a treinar a Rede Neural NARX. Todos os sinais foram gerados com uma frequência de amostragem igual a $15.360 \mathrm{~Hz}$. A frequência da componente fundamental foi definida de forma aleatória para cada evento no intervalo de $58 \mathrm{~Hz}$ a $62 \mathrm{~Hz}$. Apesar da frequência da fundamental poder variar para cada sinal no banco de dados gerado, o filtro Notch foi projetado de forma fixa para atenuação da frequência $60 \mathrm{~Hz}$, o que torna a simulação mais realística por considerar uma variação não estimada na frequência da rede. Neste caso, os sinais foram gerados com um ruído branco Gaussiano aditivo a SNRs (Signal-to-noise ratio) de $50 \mathrm{~dB}$.

A partir da implementação do método de validação cruzada do tipo $k$-fold, com $k=10,10$ redes foram treinadas de forma a estimar o próximo valor do sinal da tensão, baseando em valores anteriores da componente fundamental extraída pelo filtro Notch e do próprio sinal de tensão. O número de atrasos utilizados foram 3 ou 7 para fins de comparação da influência dessa escolha nos resultados. Além disso, o número de neurônios escondidos das redes variou de 3 a 30 . Para treinamento das redes foi utilizado backpropagation com algoritmo de otimização de Levenberg-Marquardt com regularização Bayesiana e 2.000 épocas de treino. A partir do treino das 10 redes NARX foi construído um ensemble composto por todas em que o erro final do detector é obtido a partir média da saída de todas as redes. Assim, é realizada a análise do resíduo do ensemble, sendo que um distúrbio é detectado no instante em que o erro da rede é maior do que duas vezes e meia o desvio padrão do erro da mesma em eventos de sinais nominais de tensão.

O detector proposto neste trabalho foi avaliado em seis diferentes tipos de distúrbios em sinais sintéticos de tensão, além do próprio sinal nominal, quais sejam: Notch, Harmônico, Transitório Oscilatório (capacitivo), Transitório Impulsivo (spike), elevação (swell) e afundamento (sag) de tensão. As mesmas características de frequência fundamental e de amostragem dos sinais nominais apresentadas anteriormente são aplicadas aqui. Os sinais foram gerados com um ruído branco Gaussiano aditivo a SNRs de $40 \mathrm{~dB}$, $50 \mathrm{~dB}, 60 \mathrm{~dB}$ e $70 \mathrm{~dB}$, em um total de 1.000 eventos para cada tipo de sinal em cada SNR. De acordo com Tomic et al. (2007) sinais reais normalmente possuem SNR na faixa de 50 a $70 \mathrm{~dB}$. Importante observar que, apesar das redes serem treinadas com eventos nominais a SNR de $50 \mathrm{~dB}$, essas mesmas redes serão avaliadas em diferentes SNRs de forma a testar sua robustez ao ruído.

Portanto, os resultados podem ser separados em duas etapas:

- análise de desempenho das redes NARX neurais onde é verificada as influências do número de atrasos e da escolha do número de neurônios escondidos no erro de predição em sinais nominais;

- validação do detector na presença de sinais com distúrbios em diferentes SNRs.

\subsection{Treinamento do Detector NARX Neural}

A Fig.3 apresenta o erro médio quadrático de treino e validação do detector NARX durante o processo de validação cruzada de 10 folds. São apresentados valores de erro de acordo com o número de atrasos das entradas da rede (3 ou 7) e com a variação do número de neurônios escondidos $(3,5,10,15,20$ e 30$)$.

Como pode ser observado, principalmente em dados de treino, o aumento do número de atrasos reduziu o erro médio das redes, justificando assim esse aumento no número de entradas da rede, de seis para catorze. Com relação ao número de neurônios escondidos, a partir de 10 neurônios não foi constatada melhoria significativa no desempenho das redes que justificasse esse aumento na sua complexidade. Assim, a configuração escolhida para rede foi de 14 entradas ( 7 atrasos para cada tipo de entrada - fundamental extraída e parte auto-regressiva), com 10 neurônios escondidos. Essas serão as redes que farão parte do ensemble validado na próxima seção.

\subsection{Validação do Detector NARX Neural}

A partir dos eventos gerados com ou sem distúrbio em diferentes valores de SNR, o detector (ensemble) definido na seção anterior foi validado. As Tab. 2, 3, 4 e 5 apresentam os acertos de classificação obtidos para cada tipo de evento.

Como o objetivo é implementar um sistema que detecte o distúrbio de forma mais rápida possível, foi realizada uma análise da porcentagem de acerto à medida que as amostras do evento são processadas pelo detector. Por exemplo, na Tab. 2, quando ocorre um distúrbio do tipo 
(a)

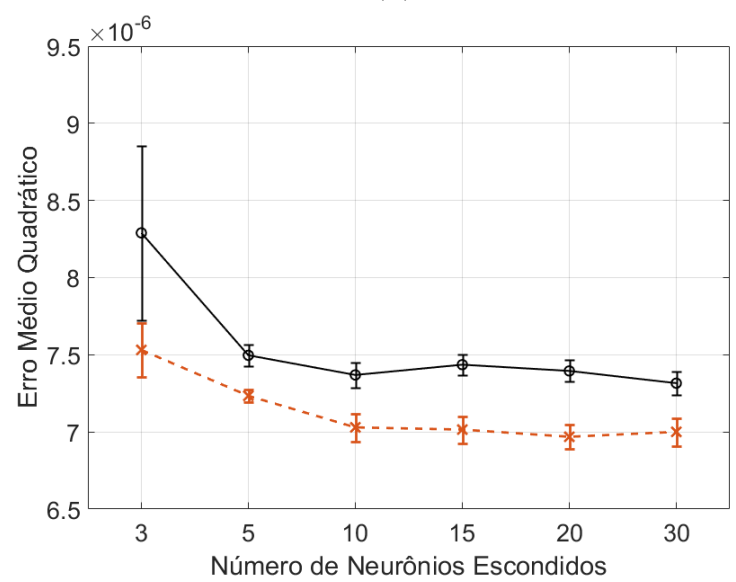

(b)

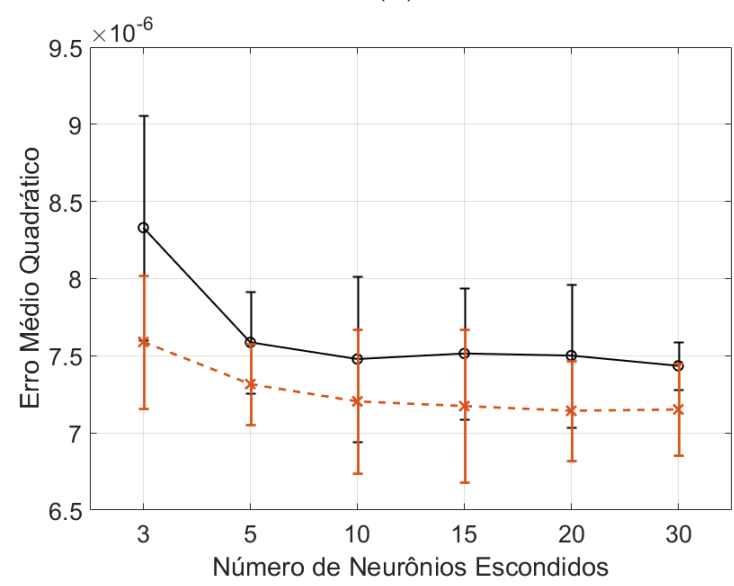

Figura 3. Erro médio quadrático obtido na execução do $k$-fold, em que as barras representam o desvio padrão, das 10 redes estimadas em (a) dados de treinamento e (b) dados de validação. Linhas contínuas em preto representam redes com 3 atrasos e linhas tracejadas em vermelho representam redes com 7 atrasos.

notch, na primeira amostra de ocorrência desse distúrbio são detectados $94,9 \%$ desses eventos. Aplicando o detector proposto na segunda amostra desses eventos, dos $5,1 \%$ que não foram detectados, $2,6 \%$ passaram a ser detectados, somando $97,5 \%$ de acerto até a segunda amostra do evento e assim por diante, até a sexta amostra.

Ao analisar essas tabelas é possível inferir que o sistema proposto detecta satisfatoriamente a maioria dos distúrbios (ou não produz falsos alarmes) nos eventos estudados, com exceção da elevação e afundamento de tensão. Boa parte dos eventos são detectados já na primeira amostra e, a partir da sexta amostra, praticamente todos os eventos foram detectados corretamente, o que comprova a eficiência do método em detectar rapidamente a ocorrência de um distúrbio. Ademais, no caso de SNR igual a $50 \mathrm{~dB}, 60 \mathrm{~dB}$ e $70 \mathrm{~dB}$ melhores resultados foram obtidos, sendo essas as relações sinal-ruído mais comum de se encontrar em redes. Para SNR igual a 40dB, harmônico, sag e swell são mais difíceis de detectar, apesar de que já na sexta amostra 92,5\% dos eventos com harmônicos foram detectados.

Pelo pior desempenho do detector em eventos com sag e swell, foi realizada uma análise do acerto do mesmo à me-
Tabela 2. Acerto em porcentagem do comitê de 10 detectores de acordo com o número de amostras de ocorrência do distúrbio para SNR igual a 40dB.

\begin{tabular}{ccccccc} 
Distúrbio & 1 & 2 & 3 & 4 & 5 & 6 \\
\hline Nenhum & 98,8 & - & - & - & - & - \\
\hline Notch & 94,9 & 97,5 & 97,5 & 97,6 & 97,6 & 99,9 \\
\hline Harm. & 70 & 75 & 77,5 & 80 & 85 & 92,5 \\
\hline Capc. & 98,6 & 100 & 100 & 100 & 100 & 100 \\
\hline Spike & 97,9 & 99,7 & 100 & 100 & 100 & 100 \\
\hline Sag & 25 & 35 & 40 & 42,5 & 45 & 52,5 \\
\hline Swell & 2,5 & 2,5 & 2,5 & 5 & 7,5 & 10 \\
\hline
\end{tabular}

Tabela 3. Acerto em porcentagem do comitê de 10 detectores de acordo com o número de amostras de ocorrência do distúrbio para SNR igual a $50 \mathrm{~dB}$.

\begin{tabular}{ccccccc} 
Distúrbio & 1 & 2 & 3 & 4 & 5 & 6 \\
\hline Nenhum & 98,7 & - & - & - & - & - \\
\hline Notch & 98,6 & 99,4 & 99,4 & 99,4 & 99,4 & 100 \\
\hline Harm. & 95 & 97,5 & 97,5 & 97,5 & 100 & 100 \\
\hline Capc. & 99,3 & 100 & 100 & 100 & 100 & 100 \\
\hline Spike & 100 & 100 & 100 & 100 & 100 & 100 \\
\hline Sag & 67,5 & 77,5 & 80 & 80 & 80 & 80 \\
\hline Swell & 17,5 & 30 & 37,5 & 45 & 45 & 45 \\
\hline
\end{tabular}

Tabela 4. Acerto em porcentagem do comitê de 10 detectores de acordo com o número de amostras de ocorrência do distúrbio para SNR igual a $60 \mathrm{~dB}$.

\begin{tabular}{ccccccc} 
Distúrbio & 1 & 2 & 3 & 4 & 5 & 6 \\
\hline Nenhum & 98,5 & - & - & - & - & - \\
\hline Notch & 98,9 & 99,4 & 99,4 & 99,4 & 99,4 & 100 \\
\hline Harm. & 97,5 & 100 & 100 & 100 & 100 & 100 \\
\hline Capc. & 100 & 100 & 100 & 100 & 100 & 100 \\
\hline Spike & 100 & 100 & 100 & 100 & 100 & 100 \\
\hline Sag & 90 & 95 & 95 & 95 & 95 & 95 \\
\hline Swell & 35 & 45 & 50 & 55 & 57,5 & 57,5 \\
\hline
\end{tabular}

Tabela 5. Acerto em porcentagem do comitê de 10 detectores de acordo com o número de amostras de ocorrência do distúrbio para SNR igual a $70 \mathrm{~dB}$.

\begin{tabular}{ccccccc} 
Distúrbio & 1 & 2 & 3 & 4 & 5 & 6 \\
\hline Nenhum & 98,1 & - & - & - & - & - \\
\hline Notch & 99,2 & 99,5 & 99,5 & 99,5 & 99,5 & 100 \\
\hline Harm. & 95 & 100 & 100 & 100 & 100 & 100 \\
\hline Capc. & 100 & 100 & 100 & 100 & 100 & 100 \\
\hline Spike & 100 & 100 & 100 & 100 & 100 & 100 \\
\hline Sag & 87,5 & 87,5 & 87,5 & 87,5 & 90 & 92,5 \\
\hline Swell & 55,0 & 57,5 & 57,5 & 60 & 60 & 62,5 \\
\hline
\end{tabular}

dida que o número de amostras processadas aumentasse. Esse resultado é apresentado na Fig. 4. Como pode ser observado, o índice de acerto é realmente baixo em ambos distúrbios na primeira amostra, mas, a partir da vigésima amostra, todos os eventos com sag foram detectados, o mesmo ocorreu com eventos swell a partir da quadragésima amostra. Considerando que um ciclo do sinal possui 256 amostras, a detecção em menos de um quarto do ciclo é ainda bastante interessante. 


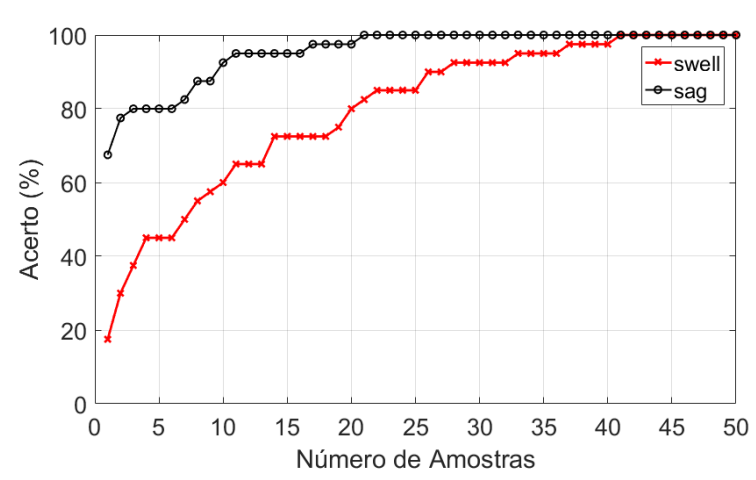

Figura 4. Acerto do detector em padrões com sag e swell em função do número de amostras desde o início da ocorrência do distúrbio (SNR igual a $50 \mathrm{~dB}$ ).

\section{CONCLUSÃO}

Neste trabalho foi proposto um detector de distúrbios de qualidade de energia baseado em modelos NARX neurais. A partir da análise de resíduos de tais modelos foi possível detectar diferentes tipos de distúrbios de uma forma muito rápida, utilizando poucas amostras do sinal de tensão.

O sistema proposto foi analisado em situações em que a frequência da componente fundamental do sinal variava dentro de um intervalo $(58 \mathrm{~Hz}-62 \mathrm{~Hz})$ e com diferentes relações sinal-ruído $(40 \mathrm{~dB}, 50 \mathrm{~dB}, 60 \mathrm{~dB}$ e $70 \mathrm{db})$. Com exceção da elevação e afundamento de tensão, em todos os outros distúrbios e sinal nominal, a abordagem apresentada obteve resultados satisfatórios ao analisar até a sexta amostra de ocorrência do distúrbio. No caso de afundamento e elevação de tensão foi observado que depois de 20 e 40 amostras, respectivamente, a detecção atingiu seu valor máximo, sendo ainda considerada uma detecção rápida por ser necessário menos que um quarto do ciclo para detecção.

Como trabalhos futuros espera-se estudar outras configurações de redes como as redes recorrentes e desenvolver modelos NARX para a classificação dos distúrbios.

\section{AGRADECIMENTOS}

Os autores gostariam de agradecer à FAPEMIG e ao CNPq pelo suporte financeiro.

\section{REFERÊNCIAS}

Achlerkar, P.D., Samantaray, S.R., and Sabarimalai Manikandan, M. (2018). Variational mode decomposition and decision tree based detection and classification of power quality disturbances in grid-connected distributed generation system. IEEE Transactions on Smart Grid, 9(4), 3122-3132.

Barbosa, B.H.G. and Ferreira, D.D. (2013). Classification of multiple and single power quality disturbances using a decision tree-based approach. Journal of Control, Automation and Electrical Systems, 24(5), 638-648.

Bollen, M.H. and Gu, I.Y. (2006). Signal processing of power quality disturbances, volume 30 . John Wiley \& Sons.

Brown, R.E. (2008). Impact of smart grid on distribution system design. In 2008 IEEE Power and Energy Society
General Meeting - Conversion and Delivery of Electrical Energy in the 21st Century, 1-4. doi:10.1109/PES.2008. 4596843.

Ghosh, A. and Ledwich, G. (2012). Power quality enhancement using custom power devices. Springer Science \& Business Media.

IEC TR 61000-2-1(1990) (1990). Electromagnetic environment for low-frequency conducted disturbances and signalling in public power supply systems. Standard, International Electro technical Communication, Genebra, Suíca.

IEEE Standards Board (1995). Ieee recommended practice for monitoring electric power quality. IEEE Std 1159$1995,1$.

Kamble, S. and Dupare, I. (2014). Detection of power quality disturbances using wavelet transform and artificial neural network. In 2014 Annual International Conference on Emerging Research Areas: Magnetics, Machines and Drives (AICERA/iCMMD), 1-5.

Mahela, O.P., Shaik, A.G., and Gupta, N. (2015). A critical review of detection and classification of power quality events. Renewable and Sustainable Energy Reviews, 41, $495-505$.

Mendes, T.M., Ferreira, D.D., da Silva, J.P., Barbosa, B.H., and Duque, C.A. (2015). Monitoramento multidimensional de qualidade de energia elétrica para smart grids. In Anais do XI CBQEE-Conferência Brasileira sobre Qualidade da Energia Elétrica, 1-4.

Mitra, S.K. and Kuo, Y. (2006). Digital signal processing: a computer-based approach, volume 2. McGraw-Hill New York.

Nagata, E.A., Ferreira, D.D., Duque, C.A., and Cequeira, A.S. (2018). Voltage sag and swell detection and segmentation based on independent component analysis. Electric Power Systems Research, 155, $274-280$.

Ribeiro, E.G., Mendes, T.M., Dias, G.L., Faria, E.R., Viana, F.M., Barbosa, B.H., and Ferreira, D.D. (2018). Real-time system for automatic detection and classification of single and multiple power quality disturbances. Measurement, 128, 276 - 283.

Ribeiro, M.V. and Pereira, J.L.R. (2007). Classification of single and multiple disturbances in electric signals. EURASIP Journal on Advances in Signal Processing, (2), 1.

Siegelmann, H.T., Horne, B.G., and Giles, C.L. (1997). Computational capabilities of recurrent narx neural networks. IEEE Transactions on Systems, Man, and Cybernetics, Part B (Cybernetics), 27(2), 208-215. doi: 10.1109/3477.558801.

Thirumala, K., Prasad, M.S., Jain, T., and Umarikar, A.C. (2018). Tunable-q wavelet transform and dual multiclass svm for online automatic detection of power quality disturbances. IEEE Transactions on Smart Grid, 9(4), 3018-3028.

Tomic, J.J., Kusljevic, M.D., and Vujicic, V.V. (2007). A new power system digital harmonic analyzer. IEEE Transactions on Power Delivery, 22(2), 772-780.

Ward, D.J. et al. (1990). Power quality-two different perspectives. IEEE Transactions on Power Delivery, 5(3), 1501-1513. doi:10.1109/61.57994. 